\title{
ANALYSIS OF AXIALLY MAGNETIZED PERMANENT MAGNET BEARING CHARACTERISTICS
}

\author{
S. I. Bekinal ${ }^{1}$ and T. R. Anil ${ }^{2}$ \\ Department of Mechanical Engineering, Gogte Institute of Technology, Belgaum- \\ 590008, India. 'Corresponding author, Email: sibekinal@git.edu, \\ ${ }^{2}$ Email:tranil@git.edu
}

\author{
S. Jana ${ }^{3}$ \\ ${ }^{3}$ Propulsion Division, National Aerospace Laboratories, Bangalore-560017, India. \\ Email:sjana@nal.res.in
}

\begin{abstract}
The use of permanent magnets as bearings has gained the attention of researchers nowadays. The characteristics of forces and moments have to be analysed thoroughly for the proper design of permanent magnet bearings. This paper presents a mathematical model of an axially magnetized permanent magnet bearing (ring magnets) using Coulombian model and a vector approach to estimate the force, moment and stiffness. A MATLAB code is developed for evaluating the parameters for five degrees of freedom (three translational and two rotational) of the rotor. Furthermore, it is extended to analyse stacked ring magnets with alternate axial polarization. The proposed model is validated with the available literature. Comparison of force and stiffness results of the presented model with the results of three dimensional (3D) finite element analysis using ANSYS shows good agreement. Finally, the cross coupled stiffness values in addition to the principal stiffness values are presented for elementary structures and also for stacked structures with three ring permanent magnets.
\end{abstract}

Keywords: Coulombian model, force, moment, permanent magnet, stiffness, vector approach

\section{INTRODUCTION}

Permanent magnet bearings are contact free bearings wherein the rotor is levitated using an attractive or repulsive forces generated between the magnets. The contact free feature of permanent magnet bearings offers attractive advantages like friction free and lubrication free operation, low maintenance, long life etc. Exhaustive work is carried out by Yonnet [1,2], Delamare [3] for synthesizing different configurations of the magnetic bearings. These are used in many applications such as flywheels, turbo molecular pumps, turbomachines and conveyor systems [4-7]. It is proposed that the force and stiffness of the permanent magnet bearing can be increased if layers of rings are stacked with specific magnetization pattern [8]. The analytical equations for calculating the magnetic field [9-14], force and stiffness [15-20] in bearings for both axial and radial magnetization are widely available in the recent past. However in these works, the ring magnets are concentric, which might not be prevailing in 
actual scenario. The expressions for evaluating the moments on the rotor in an axially polarized bearings are presented by Jiang et al. [21, 22]. These analytical expressions involve elliptical integrals which are tedious to deal. The present work focuses on the mathematical treatment for evaluating force, moment and stiffness characteristics of a permanent magnet bearing considering three translational ( $\mathrm{x}, \mathrm{y}$ and $\mathrm{z})$ and two angular degrees $(\xi$ and $\gamma)$ of freedom of the rotor magnet ring using a simple vector approach [23]. A MATLAB code is written for estimating the forces, moments, stiffness and cross coupled stiffnesses between the stator - rotor made of two non-concentric ring magnets and for multiple rings based on the Coulombian model. The results of mathematical model are compared with a case available in the literature [24] and with the results of 3D finite element analysis using ANSYS. Finally, a full $5 \times 5$ stiffness matrix (Eq. (1)) representing five degrees of freedom is developed for elementary structures of the bearings and stacked structure with three ring permanent magnets.

$$
K=\left[\begin{array}{ccccc}
K_{X X} & K_{X Y} & K_{X Z} & K_{X \xi} & K_{X \gamma} \\
K_{Y X} & K_{Y Y} & K_{Y Z} & K_{Y \xi} & K_{Y \gamma} \\
K_{Z X} & K_{Z Y} & K_{Z Z} & K_{Z \xi} & K_{Z \gamma} \\
K_{\xi X} & K_{\xi Y} & K_{\xi Z} & K_{\xi \xi} & K_{\xi \gamma} \\
K_{\gamma X} & K_{\gamma Y} & K_{\gamma Z} & K_{\gamma \xi} & K_{\gamma \gamma}
\end{array}\right]
$$

\section{FORCE, STIFFNESS AND MOMENT ESTIMATION}

The forces between the two ring magnets, one fixed to the rotor and the other to the stator, need to be calculated to estimate the bearing characteristics. Two different methods, namely, a dipole and surface charge density are used to calculate the forces between the two magnets. The dipole method is limited to specific cases, as this provides results for the dimensions of magnets which are equal to or less than the air gap. The latter method is used in the permanent magnet bearings where magnet dimensions are larger than the bearing rotor-stator gap, thereby utilized in the present analysis. In this method, it is assumed that the permanent magnet surface is charged with a positive and negative charge according to the direction of polarization. The forces of attraction or repulsion between the polarized surfaces of the magnets are calculated using the Coulomb's law of force. The basic configuration of a permanent magnet bearing is shown in Fig. 1.

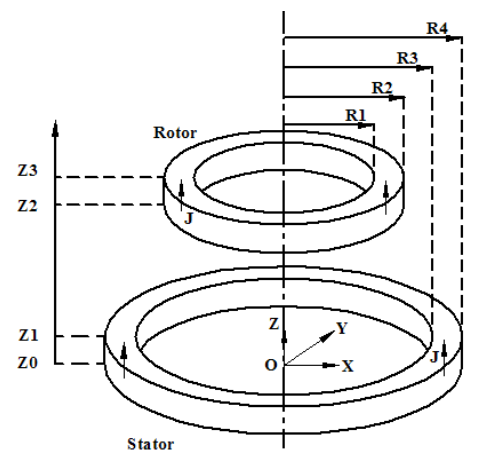

Figure 1. Configuration of permanent magnet bearing.

The inner magnet is fixed to the rotor and the stator to the outer magnet. In the 
present analysis it is assumed that the inner magnet can move in $\mathrm{x}, \mathrm{y}$ and $\mathrm{z}$ tanslational directions and $\xi$ and $\gamma$ angular directions with respect to the outer magnet. The inner and outer radii of the inner permanent magnet ring are R1 and R2 respectively. The inner and outer radii of the outer permanent magnet ring are $\mathrm{R} 3$ and $\mathrm{R} 4$ respectively. The thicknesses of the outer and inner magnets are $\mathrm{L}=\mathrm{Z} 1-\mathrm{Z} 0$ and $\mathrm{L}$ $=\mathrm{Z} 3-\mathrm{Z} 2$ respectively. Magnetic polarizations $(\mathrm{J})$ of both the magnets are in the axial direction as depicted by Fig. 1. Modeling of the bearing is carried out by knowing the forces acting on the rotor magnet which in turn can be utilized to estimate the bearing stiffness. The Coulombian model of the magnets is adopted, wherein each permanent magnet is represented by surfaces with fictitious magnetic pole surface densities in the direction of polarization. Fig. 2(a) shows the arrangement of rotor and stator magnets with magnetization in the axial direction. Surfaces $\mathrm{A}$ and $\mathrm{C}$ are the fictitious charged surfaces of rotor magnet and surfaces B and D are for the stator magnet. There are magnetic forces of attraction and repulsion between the charged surfaces of the rotor and stator magnet. The net force acting is the bearing reactions for various positions of the rotor. In literature, analytical or semi-analytical expressions are derived and used for the purpose of calculating the bearing forces and stiffness values [15-20]. The rotor magnet is displaced from its nominal position by a distance of ' $\mathbf{e}$ ' in XYZ coordinate system as shown in Fig. 2(a) and (b).

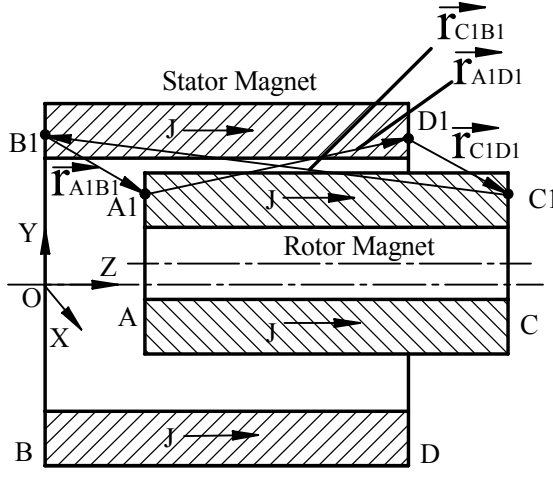

(a)

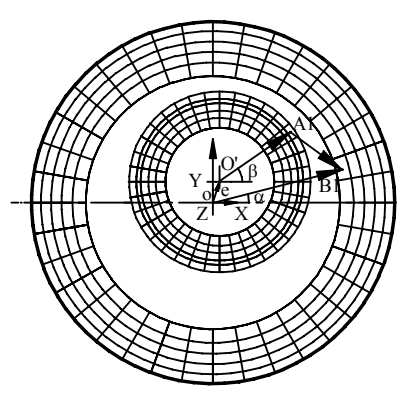

(b)

Figure 2. Force and moment calculation model. (a) Arrangement of rotor and stator magnets with respect to translational degrees of freedom (x, y and $\mathrm{z}$ ). (b) Elements.

Fig. 3 represents positions of the rotor when it is concentric with stator and with an angular displacement of ' $\xi$ ' with $\mathrm{Z}$ axis in the anticlockwise directions. 


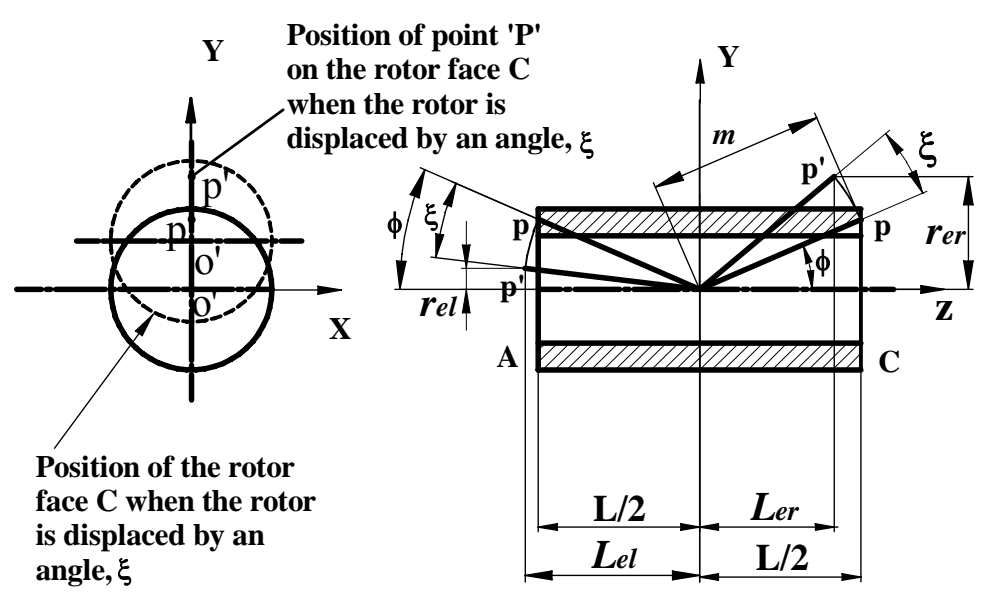

Figure 3. Positions of rotor with points ' $p$ ' and ' $p$ " on the magnet faces $A$ and $C$ when it is concentric with stator and with an angular displacement ' $\xi$ ' with $\mathrm{Z}$ axis in the anticlockwise direction.

The elemental force on discrete surface element 'A1' of the rotor magnet surface ' $A$ ' due to the surface element 'B1' on the stator magnet surface ' $B$ ' can be expressed as [25],

$$
\vec{F}_{A 1 B 1}=\frac{J^{2} S_{A 1} S_{B 1}}{4 \pi \mu_{0} r_{A 1 B 1}^{3}} \vec{r}_{A 1 B 1}
$$

where, $\mathrm{J}$ is the magnet surface flux density (equal for both the magnets), $S_{A 1}$ is the surface area of element $\mathrm{A} 1$ and $S_{B 1}$ is the surface area of element B1, $\vec{r}_{A 1 B 1}$ is the distance vector between element $\mathrm{A} 1$ and $\mathrm{B} 1, \mu_{0}$ is the absolute magnetic permeability. The vector $\vec{r}_{A 1 B 1}$ can be expressed in 'XYZ' coordinate system as,

$$
\vec{r}_{A 1 B 1}=\left(X_{A 1}-X_{B 1}\right) i+\left(Y_{A 1}-Y_{B 1}\right) j+\left(Z_{A 1}-Z_{B 1}\right) k
$$

where, $\mathbf{i}, \mathbf{j}$ and $\mathbf{k}$ are the unit vectors in $\mathrm{X}, \mathrm{Y}$, and $\mathrm{Z}$ axes, $X_{A 1}, Y_{A 1}, Z_{A 1}$ is the coordinate of element $\mathrm{A} 1$ and $X_{B 1}, Y_{B 1}, Z_{B 1}$ is the co-ordinate of the element B1. The coordinates of the discrete elements are expressed by considering the movement of the rotor magnet $\mathrm{e}=\mathrm{xi}+\mathrm{y} \mathbf{j}+\mathrm{zk}$ and the positions of the elements in terms of the mean and equivalent radii and the angles $\alpha, \beta, \phi$ and $\xi$ can be expressed as:

$$
\begin{array}{ll}
m=\sqrt{(0.5 L)^{2}+\left(r_{A 1 M} \sin (\beta)\right)^{2}} & \tan (\phi)=\frac{r_{A 1 M} \sin (\beta)}{0.5 L} \\
r_{e l}=m \sin (\phi-\xi) & r_{e r}=m \sin (\phi+\xi) \\
L_{e l}=m \cos (\phi-\xi) & L_{e r}=m \cos (\phi+\xi) \\
\vec{X}_{A 1}=\left(x+r_{e l}\right) i & \vec{X}_{B 1}=\left(x+r_{B 1 M} \cos (\alpha)\right) i \\
\vec{Y}_{A 1}=\left(y+r_{e l}\right) j & \vec{Y}_{B 1}=\left(y+r_{B 1 M} \sin (\alpha)\right) j
\end{array}
$$




$$
\vec{Z}_{A 1}=\left(z-\left(L_{e l}-0.5 L\right)\right) k \quad \vec{Z}_{B 1}=0
$$

where, $\alpha$ and $\beta$ are refered in XY plane (Fig. 2 (a) and (b)) and $\phi$ and $\xi$ are in YZ plane (Fig. 3), $r_{A 1 M}$ is the mean radius of element A1 from inner magnet centre ' $\mathrm{O}$ ', $r_{B 1 M}$ is the mean radius of element $\mathrm{B} 1$ from outer magnet centre ' $\mathrm{O}$ ', $r_{e l}$ is equivalent radius of element $\mathrm{A} 1$ from the axis of rotor magnet and $L_{e l}$ is equivalent distance of element A1 from the centre of magnet in $\mathrm{Z}$ direction. Combining Eq. (2) and (3), the elemental force in terms of components in XYZ co-ordinates can be written as,

$$
\vec{F}_{A 1 B 1}=F_{A 1 B 1 X} i+F_{A 1 B 1 Y} j+F_{A 1 B 1 Z} k
$$

In the similar fashion, elemental forces $\vec{F}_{A 1 D 1}, \vec{F}_{C 1 B 1}$ and $\vec{F}_{C 1 D 1}$ due to elements on rotor and stator magnet surfaces $\mathrm{B}, \mathrm{C}$ and $\mathrm{D}$ can be written by considering the respective vector distances (Fig. 2(a)). Considering ' $n$ ' number of discrete surface elements in the inner magnet and ' $\mathrm{m}$ ' number of discrete surface elements in the outer magnet, the resultant forces in XYZ axes, on the rotor magnet can be expressed as a summation of all the elemental forces which is presented below (Eq. (6)-(8)).

$$
\begin{gathered}
F_{X}=\sum_{p=1, q=1}^{p=n, q=m} F_{A p B q X}+\sum_{p=1, q=1}^{p=n, q=m} F_{A p D q X}+ \\
\sum_{p=1, q=1}^{p=n, q=m} F_{C p B q X}+\sum_{p=1, q=1}^{p=n, q=m} F_{C p D q X} \\
F_{Y}=\sum_{p=1, q=1}^{p=n, q=m} F_{A p B q Y}+\sum_{p=1, q=1}^{p=n, q=m} F_{A p D q Y}+ \\
\sum_{p=1, q=1}^{p=n, q=m} F_{C p B q}+\sum_{p=1, q=1}^{p=n, q=m} F_{C p D q Y} \\
F_{Z}=\sum_{p=1, q=1}^{p=n, q=m} F_{A p B q Z}+\sum_{p=1, q=1}^{p=n, q=m} F_{A p D q Z}^{+} \\
\sum_{p=1, q=1}^{p=n, q=m} F_{C p B q Z}+\sum_{p=1, q=1}^{p=n, q=m} F_{C p D q Z}
\end{gathered}
$$

The stiffness of the bearing in Cartesian coordinate system can be obtained by the numerical differentiation scheme, once the resultant forces are computed. A three point midpoint formula for differentiation is used to obtain stiffness values in radial, axial and angular directions. The principal radial stiffness exerted between two ring permanent magnets along $\mathrm{X}$ direction at $x$ can be expressed as follows:

$$
K_{X X}=\frac{d F_{X}}{d X}=\frac{1}{2 \Delta x}\left[F_{X}(x+\Delta x)-F_{X}(x-\Delta x)\right]-\frac{\Delta x^{2}}{6} F_{X}^{3}(\xi)
$$

where $\xi$ lies between $(x-\Delta x)$ and $(x+\Delta x)$ and for smaller values of $\Delta x$, the Eq. (9) can be expressed as: 


$$
K_{X X} \approx \frac{d F_{X}}{d X}=\frac{1}{2 \Delta x}\left[F_{X}(x+\Delta x)-F_{X}(x-\Delta x)\right]
$$

Similarly, principal radial stiffness along $\mathrm{Y}$ and principal axial stiffness in $\mathrm{Z}$ direction can be written as follows (Eq. (11) and (12)):

$$
\begin{aligned}
& K_{Y Y} \approx \frac{d F_{Y}}{d Y}=\frac{1}{2 \Delta y}\left[F_{Y}(y+\Delta y)-F_{Y}(y-\Delta y)\right] \\
& K_{Z Z} \approx \frac{d F_{Z}}{d Z}=\frac{1}{2 \Delta z}\left[F_{Z}(z+\Delta z)-F_{Z}(z-\Delta z)\right]
\end{aligned}
$$

Cross coupled radial and axial-radial stiffnesses can be expressed as (Eq. (13)-15):

$$
\begin{aligned}
& K_{X Y}=K_{Y X} \approx \frac{d F_{X}}{d Y}=\frac{1}{2 \Delta y}\left[F_{X}(x+\Delta x)-F_{X}(x-\Delta x)\right] \\
& K_{X Z}=K_{Z X} \approx \frac{d F_{X}}{d Z}=\frac{1}{2 \Delta z}\left[F_{X}(x+\Delta x)-F_{X}(x-\Delta x)\right] \\
& K_{Y Z}=K_{Z Y} \approx \frac{d F_{Z}}{d Y}=\frac{1}{2 \Delta y}\left[F_{Z}(z+\Delta z)-F_{Z}(z-\Delta z)\right]
\end{aligned}
$$

Movements inside the bearing necessiates estimation of moment acting on the rotor. In practical cases the rotor magnet movement can be assumed as a rigid body movement, thus the moment of the forces acting on the rotor magnet calculated about its centre of gravity (geometric centre for axisymmetric and isotropic magnets) provides useful information about the dynamics of the rotor magnet. The moment due to elemental force $\vec{F}_{A 1 B 1}$ about the centre of gravity of the inner magnet can be written as,

$$
\begin{aligned}
& M_{A 1 B 1 X}=F_{A 1 B 1 Y} \times L_{e l}+F_{A 1 B 1 Z} \times r_{e l} \\
& M_{A 1 B 1 Y}=F_{A 1 B 1 X} \times L_{e l}-F_{A 1 B 1 Z} \times r_{e l} \\
& M_{A 1 B 1 Z}=-F_{A 1 B 1 X} \times r_{e l}+F_{A 1 B 1 Y} \times r_{e l}
\end{aligned}
$$

In the similar fashion, the moment due to elemental forces $\vec{F}_{A 1 D 1}, \vec{F}_{C 1 B 1}$ and $\vec{F}_{C 1 D 1}$ about the centre of gravity of the rotor magnet can be written by following the proper sign conention.

The net moment acting on the rotor magnet are expressed using Eq. (16) as, 


$$
\begin{gathered}
M_{X}=\sum_{p=1, q=1}^{p=n, q=m} M_{A p B q X}+\sum_{p=1, q=1}^{p=n, q=m} M_{A p D q X}+ \\
\sum_{p=1, q=1}^{p=n, q=m} M_{C p B q X}+\sum_{p=1, q=1}^{p=n, q=m} M_{C p D q X} \\
M_{Y}=\sum_{p=1, q=1}^{p=n, q=m} M_{A p B q Y}+\sum_{p=1, q=1}^{p=n, q=m} M_{A p D q Y^{+}}+ \\
\sum_{p=1, q=1}^{p=n, q=m} M_{C p B q Y}+\sum_{p=1, q=1}^{p=n, q=m} M_{C p D q Y} \\
M_{Z}=\sum_{p=1, q=1}^{p=n, q=m} M_{A p B q Z}+\sum_{p=1, q=1}^{p=n, q=m} M_{A p D q Z}+ \\
\sum_{p=1, q=1}^{p=n, q=m} M_{C p B q Z}+\sum_{p=1, q=1}^{p=n, q=m} M_{C p D q Z}
\end{gathered}
$$

The principal moment stiffnesses exerted between the two ring permanent magnets can be expressed as

$$
K_{\xi \xi}=K_{\gamma \gamma} \approx \frac{d M_{\xi}}{d \xi}=\frac{1}{2 \Delta \xi}\left[M_{\xi}(\xi+\Delta \xi)-M_{\xi}(\xi-\Delta \xi)\right]
$$

The cross coupled angular, axial-angular and radial-angular stiffnesses can be expressed as (Eq. (21)-(24)):

$$
\begin{array}{r}
K_{\xi \gamma}=K_{\gamma \xi} \approx \frac{d M_{\xi}}{d \gamma}=\frac{1}{2 \Delta \gamma}\left[M_{\xi}(\xi+\Delta \xi)-M_{\xi}(\xi-\Delta \xi)\right] \\
K_{\xi Z}=K_{Z \xi} \approx \frac{d M_{Z}}{d \xi}=\frac{1}{2 \Delta \xi}\left[M_{Z}(Z+\Delta Z)-M_{Z}(Z-\Delta Z)\right] \\
K_{\xi X}=K_{X \xi}=K_{Y \gamma}=K_{\gamma Y} \approx \frac{d M_{\xi}}{d X}=\frac{1}{2 \Delta X}\left[M_{\xi}(\xi+\Delta \xi)-M_{\xi}(\xi-\Delta \xi)\right] \\
K_{\xi Y}=K_{Y \xi} \approx \frac{d M_{\xi}}{d Y}=\frac{1}{2 \Delta Y}\left[M_{\xi}(\xi+\Delta \xi)-M_{\xi}(\xi-\Delta \xi)\right]
\end{array}
$$

The forces and moment acting on the rotor magnet as well as the radial, axial and moment stiffness of the bearing for two different configurations are calculated and presented in the following section. This mathematical model can be used for different configurations of permanent magnet bearings made of axially magnetized ring magnets.

\section{VALIDATION OF PROPOSED MODEL}

The proposed mathematical model is used to determine the axial force and stiffness of an axially magnetized permanent magnet bearing shown as Fig. 1. Geometrical parameters considered for the analysis are presented in Table 1.

Table 1. Dimensions of the configuration.

\begin{tabular}{|l|l|l|}
\hline & Inner ring & Outer ring \\
\hline
\end{tabular}




\begin{tabular}{|c|c|c|}
\hline & dimensions & dimensions \\
\hline Inner radius $[\mathrm{mm}]$ & $\mathrm{R} 1=10$ & $\mathrm{R} 3=22$ \\
\hline Outer radius $[\mathrm{mm}]$ & $\mathrm{R} 2=20$ & $\mathrm{R} 4=32$ \\
\hline Thickness, $\mathrm{L}[\mathrm{mm}]$ & $\mathrm{Z} 1-\mathrm{Z} 0=10$ & $\mathrm{Z} 3-\mathrm{Z} 2=10$ \\
\hline Flux density $[\mathrm{T}]$ & $\mathrm{J} 1=1$ & $\mathrm{~J} 2=1$ \\
\hline
\end{tabular}

The inner magnet is placed in such a way that its magnetization direction is same as that of the stator magnet. This configuration produces positive radial stiffness; however, axial stiffness becomes negative. The axial force and stiffness values along with the results of [24] are graphed in Fig. 4. Table 2 presents a comparison of the proposed model with that reported in R. Ravaud and G. Lemarquand [24]. MATLAB code developed for the mathematical model includes the calculation of forces, stiffnesses and moments in axial, radial and angular directions, so computational cost is $0.7 \mathrm{~s}$ for a bearing made of two ring permanent magnets for one iteration. Compuational cost of the code is too low as compared to evaluating the parameters using Finite Element Analysis tools like ANSYS, COMSOL, MAXWELL 3D etc.

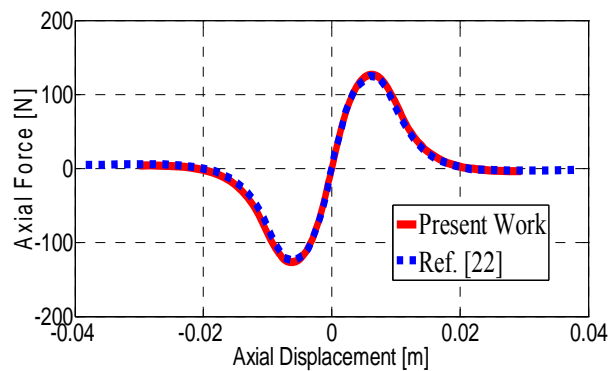

(a)

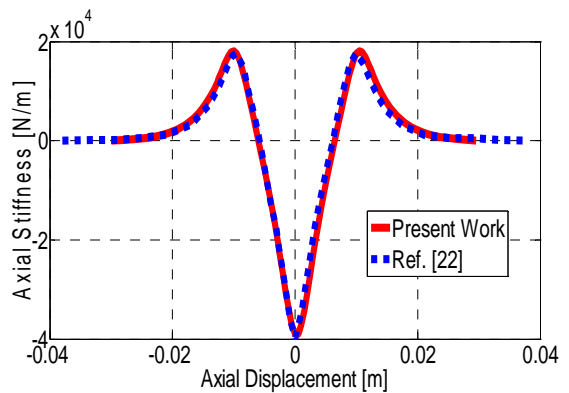

(b)

Figure 4. Characteristics of permanent magnet bearing. (a) Axial Force. (b) Axial Stiffness.

Table 2. Comparison of Results.

\begin{tabular}{|c|c|c|c|}
\hline & $\begin{array}{c}\text { Results of } \\
\text { present work }\end{array}$ & $\begin{array}{c}\text { Results of } \\
\text { R. Ravaud et al. } \\
{[24]}\end{array}$ & Variation (\%) \\
\hline Axial Force $[\mathrm{N}]$ & 126.59 & 125 & 1.2 \\
\hline $\begin{array}{c}\text { Axial Stiffness }\left|K_{Z}\right| \\
{[\mathrm{N} / \mathrm{m}]}\end{array}$ & 38881 & 39848 & 2.48 \\
\hline
\end{tabular}

It can be observed from Fig. 4 and Table 2 that, the evaluated axial force and stiffness values match very closely with that of reported analytical results for the same bearing configuration. A finite element model was built in ANSYS to validate the results of mathematical model. The model is analysed with linear brick 8488 elements and 2090 nodes (Fig. 5(a)). The properties of the magnetic material considered for the analysis are: $\mathrm{B}_{\mathrm{r}}=1.05 \mathrm{~T}, \mathrm{H}_{\mathrm{c}}=796 \mathrm{kA} / \mathrm{m}$ and $\mu_{\mathrm{r}}=1.049$. A magneto static solver was used to compute the distribution of magnetic field. The Maxwell stress tensor was used for integrating the surface of the inner ring magnet to compute 
the force in ANSYS (Fig. 5(b)). The force exerted by the stator ring on the rotor is plotted for its different axial positions in Fig. 6.

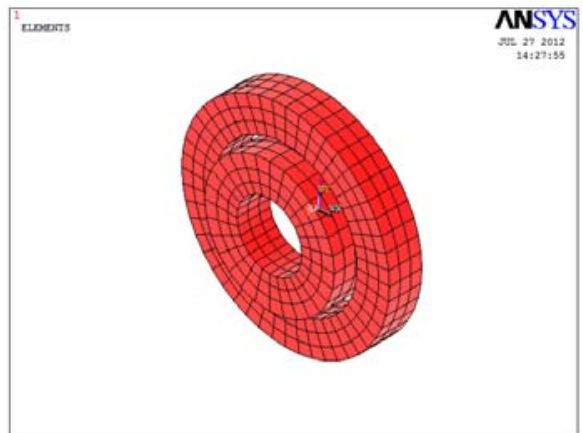

(a)

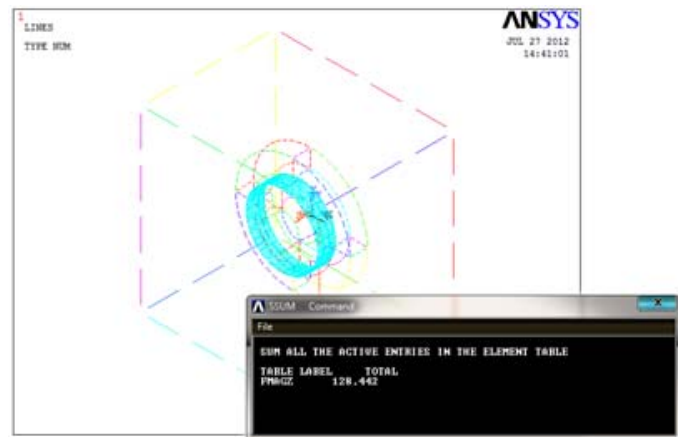

(b)

Figure 5. Results of finite element analysis in ANSYS. (a) Finite element model of ring magnets. (b) Force exerted on the inner ring.

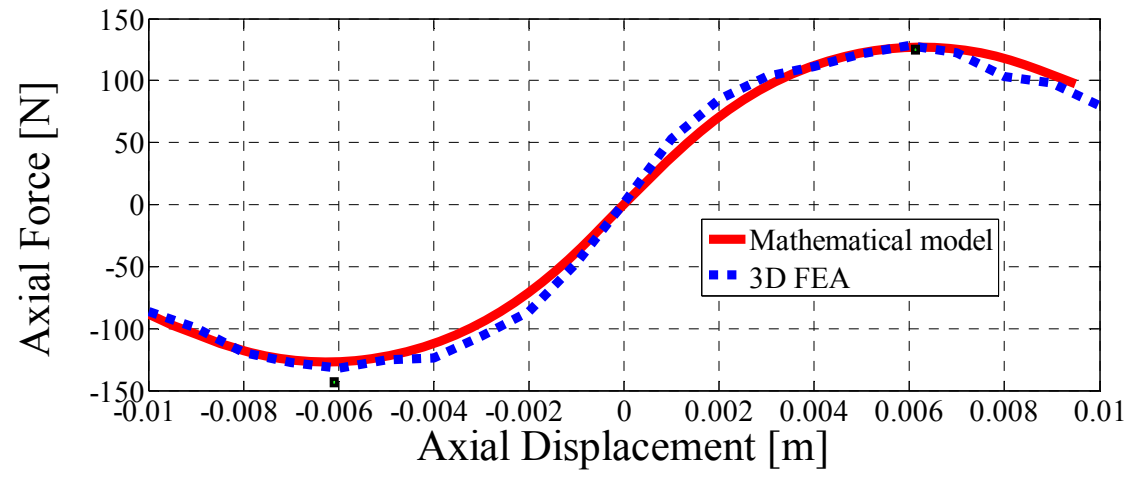

Figure 6. Axial Force Comparison.

It can be observed from Fig. 6 that not only the results of axial force obtained with 3D FEA match very closely with the results of mathematical model, but two methods also predict the same optimal point. The mismatch between the results from the mathematical model and those from FEA is less than $4 \%$.

\section{ANALYSIS OF AXIALLY MAGNETIZED BEARING CONFIGURATIONS}

\subsection{Elementary Structures}

Two elementary configurations, as shown in Fig. 7 are analysed for force, moment and stiffness parameters. The configuration in Fig. 7(a) is suitable for radial bearing application developing positive radial stiffness having negative axial stiffness. The configuration presented in Fig. 7(b) is suitable for axial bearing application (positive axial stiffness and negative radial stiffness). 


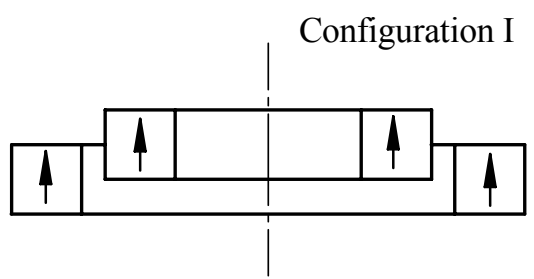

(a)

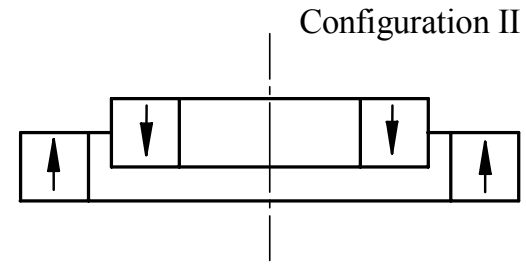

(b)

Figure 7. Configurations of permanent magnet bearing. (a) Polarization in the same direction. (b) Polarization in the opposite direction.

The axial force and stiffness computed for various axial positions of the rotor magnet in the configuration I and II are presented in Fig. 8 and Fig. 9 respectively. The axial force and stiffness are calculated at different radial offset values $(0.5,0.75$, and $1 \mathrm{~mm}$ ) of the rotor magnet in the positive $\mathrm{X}$ direction.

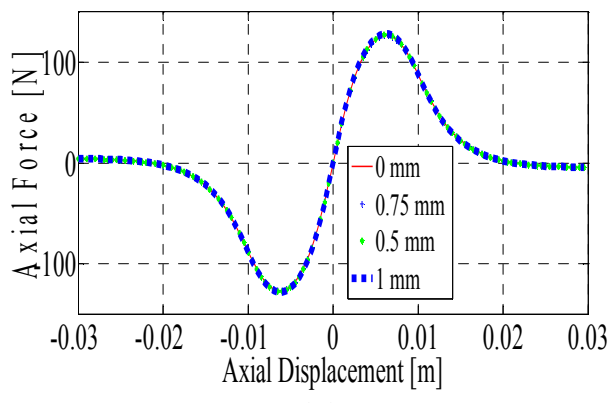

(a)

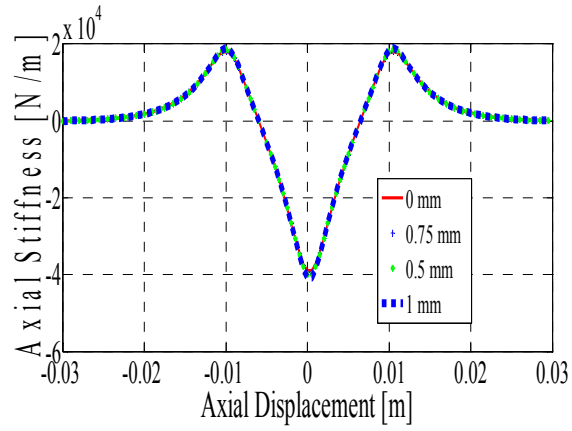

(b)

Figure 8. Characteristics of configuration I. (a) Axial force. (b) Axial stiffness.

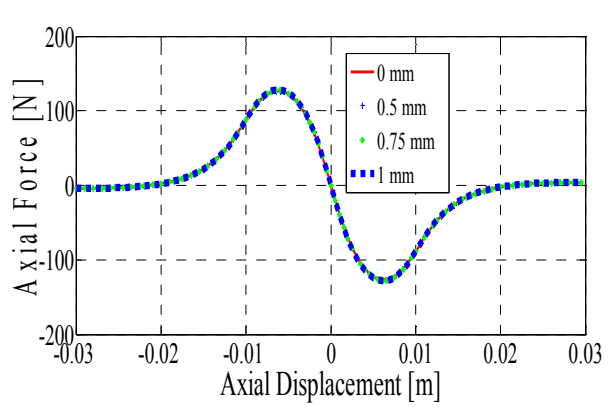

(a)

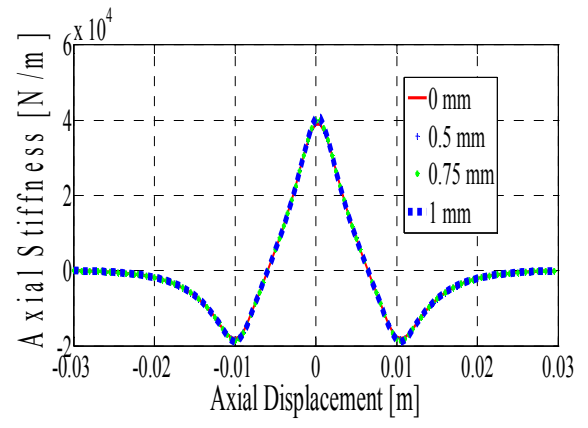

(b)

Figure 9. Characteristics of configuration II. (a) Axial force. (b) Axial stiffness.

It can be observed from the results that there is a least influence of radial offset on the axial force and stiffness values.

The moment acting on the rotor magnet as a result of radial displacements $(0.5$, 0.75 and $1 \mathrm{~mm}$ ) are calculated as a function of various axial positions of the rotor for configurations I and II and are plotted in Fig. 10. 


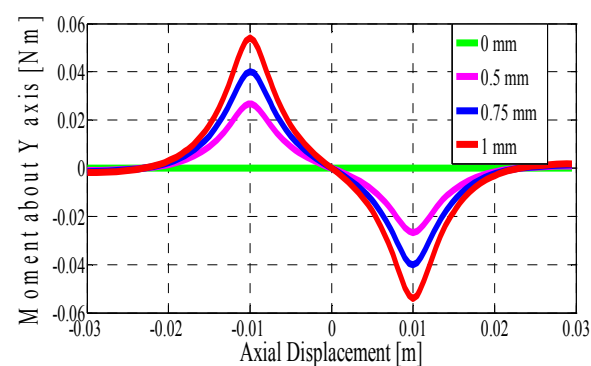

(a)

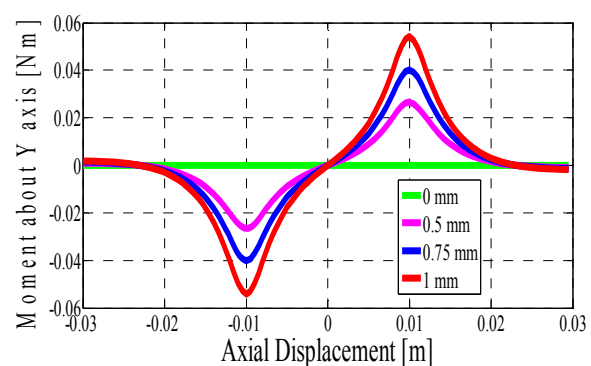

(b)

Figure 10. Moment about $\mathrm{Y}$-axis at various radial displacements in $\mathrm{X}$ axis. (a) Configuration I. (b) Configuration II.

It has been observed from analysis that the radial displacement of the rotor in $\mathrm{X}$ axis generates moment about the $\mathrm{Y}$ axis of the rotor magnet and vice-versa. The resultant moment about the $\mathrm{Z}$ axis of the rotor magnet is zero. The results show that the magnitudes of moment increase with the higher radial offset value in both the configurations and they vanish when the inner magnet is concentric with the outer magnet. Direction of the moment changes according to the magnetization direction of the inner magnet with respect to the outer magnet.

The radial forces and stiffness values of both the bearing configuration are calculated as a function of radial displacement and are presented in Fig. 11.

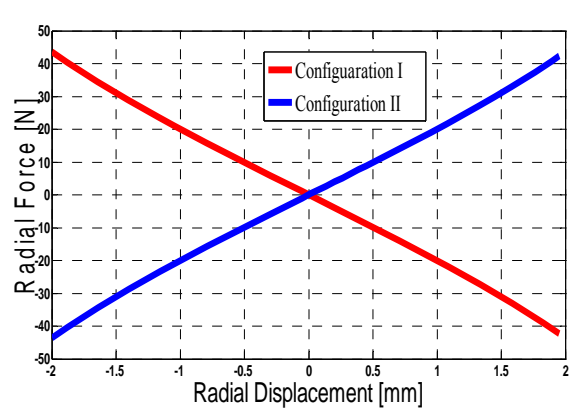

(a)

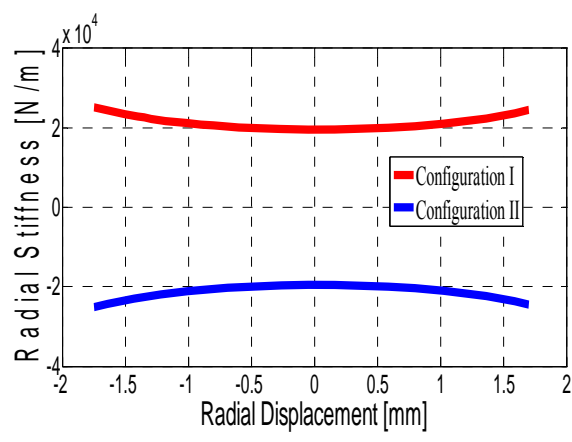

(b)

Figure 11. Characteristics of configuration I and II. (a) Radial force. (b) Radial stiffness.

It can be observed from the Fig. 11 that bearing configuration I produce positive radial stiffness and configuration II generates negative radial stiffness showing a similar trend as cited by earlier researcher [3]. Full $5 \times 5$ stiffness matrix at an axial offset of $2.8 \mathrm{~mm}$ for the configuration I is presented in Table 3 and results of configuration II are obtained by changing the sign of respective cell values.

Table 3. Results of configuration I.

\begin{tabular}{|l|l|l|l|l|l|}
\hline & $\mathrm{X}$ & $\mathrm{Y}$ & $\mathrm{Z}$ & $\xi$ & $\gamma$ \\
\hline
\end{tabular}




\begin{tabular}{|c|c|c|c|c|c|}
\hline $\mathrm{X}$ & $11.3 \mathrm{~N} / \mathrm{mm}$ & 0 & $*$ & 0 & 0 \\
\hline $\mathrm{Y}$ & 0 & $11.3 \mathrm{~N} / \mathrm{mm}$ & $* *$ & 0 & 0 \\
\hline $\mathrm{Z}$ & $*$ & $* *$ & $-22.61 \mathrm{~N} / \mathrm{mm}$ & $\#$ & $\# \#$ \\
\hline$\xi$ & 0 & 0 & $\#$ & $-1.15 \mathrm{Nm} / \mathrm{rad}$ & 0 \\
\hline$\gamma$ & 0 & 0 & $\# \#$ & 0 & $-1.15 \mathrm{Nm} / \mathrm{rad}$ \\
\hline
\end{tabular}

$$
\begin{aligned}
& \text { where } *=-2.03 \mathrm{~N} / \mathrm{mm} \text { at } \mathrm{x}=0.5 \mathrm{~mm} \\
& =-3.99 \mathrm{~N} / \mathrm{mm} \text { at } \mathrm{x}=1.0 \mathrm{~mm} \\
& \#=179.8 \mathrm{Nm} / \mathrm{rad} \text { at } \xi=10^{0} \\
& =-5.98 \mathrm{~N} / \mathrm{mm} \text { at } \mathrm{x}=1.5 \mathrm{~mm}, \quad=195 \mathrm{Nm} / \mathrm{rad} \text { at } \xi=20^{\circ} \\
& * *=-2.03 \mathrm{~N} / \mathrm{mm} \text { at } \mathrm{y}=0.5 \mathrm{~mm} \\
& =-3.99 \mathrm{~N} / \mathrm{mm} \text { at } \mathrm{y}=1.0 \mathrm{~mm} \\
& =-5.98 \mathrm{~N} / \mathrm{mm} \text { at } \mathrm{y}=1.5 \mathrm{~mm} \text {, } \\
& \text { \#\#=179.8 Nm/rad at } \gamma=10^{0} \\
& =191.8 \mathrm{Nm} / \mathrm{rad} \text { at } \gamma=15^{0} \\
& =195 \mathrm{Nm} / \mathrm{rad} \text { at } \gamma=20^{0} \text {. }
\end{aligned}
$$

\subsection{Stacked Structures With Three Ring Permanent Magnets}

The configuration composed of three ring pairs with alternate axial polarizations is presented in the Fig. 12. The variation of axial force and stiffness of the configuration with radial offset for different axial positions of the rotor is depicted by Fig. 13.

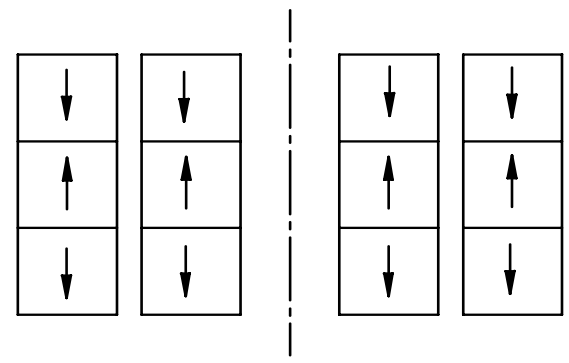

Figure 12. Cross- section view of a permanent magnet bearing composed of three ring pairs with alternate axial polarizations: $\mathrm{R} 1=10 \mathrm{~mm}, \mathrm{R} 2=20 \mathrm{~mm}, \mathrm{R} 3=22 \mathrm{~mm}$, $\mathrm{R} 4=32 \mathrm{~mm}, \mathrm{~J}=1 \mathrm{~T}$, the height of each ring permanent magnet $=10 \mathrm{~mm}$.

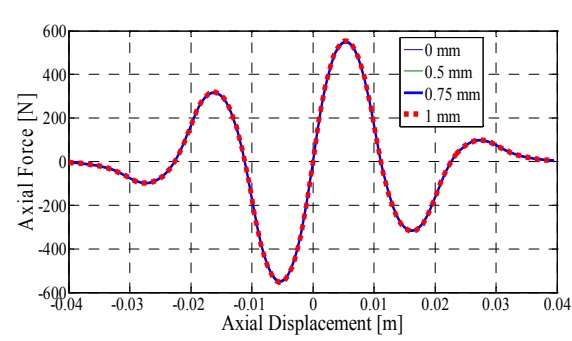

(a)

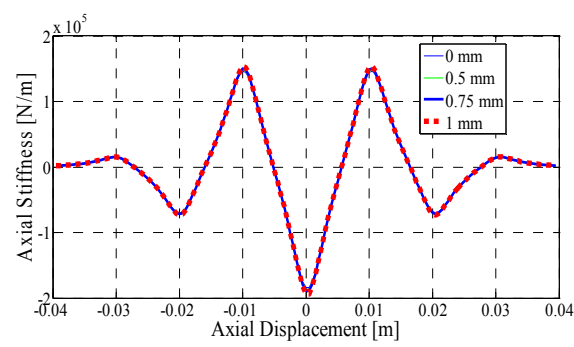

(b)

Figure 13. Characteristics of permanent magnet bearing made of three ring pairs. (a) Axial force. (b) Axial Stiffness.

The proposed mathematical approach leads to following observations:

- The maximum axial force exerted by the outer rings on the inner rings $=544 \mathrm{~N}$ and maximum axial stiffness, $\left|K_{Z}\right|=183990 \mathrm{~N} / \mathrm{m}$ (for concentric rings).

- For a radial offset of $1 \mathrm{~mm}$ in $\mathrm{X}$ direction, maximum force $=552.85 \mathrm{~N}$ and 
Stiffness, $\left|K_{Z}\right|=193300 \mathrm{~N} / \mathrm{m}$.

Negative axial stiffness implies, suitability for radial bearing application. The moment acting on the rotor magnet as the result of radial displacements are plotted in Fig. 14. The maximum moment acting on the rotor about $\mathrm{Y}$ axis is estimated as $0.4 \mathrm{Nm}$ for a radial offset of $1 \mathrm{~mm}$ in $\mathrm{X}$ direction.

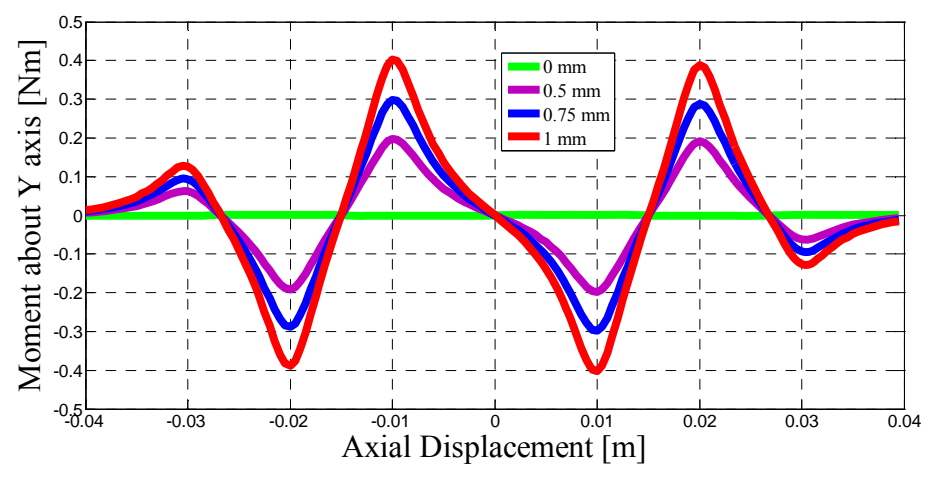

Figure 14. Moment about $\mathrm{Y}$-axis at various radial displacements in $\mathrm{X}$ axis.

A full $5 \times 5$ stiffness matrix at an axial offset of $3.0 \mathrm{~mm}$ for the configuration shown in Fig. 12 is presented in Table 5.

Table 5. Results of configuration composed of three ring pairs with alternate axial polarizations (Fig. 11).

\begin{tabular}{|c|c|c|c|c|c|}
\hline & $\mathrm{X}$ & $\mathrm{Y}$ & $\mathrm{Z}$ & $\xi$ & $\gamma$ \\
\hline $\mathrm{X}$ & $49.73 \mathrm{~N} / \mathrm{mm}$ & 0 & $*$ & 0 & 0 \\
\hline $\mathrm{Y}$ & 0 & $49.73 \mathrm{~N} / \mathrm{mm}$ & $* *$ & 0 & 0 \\
\hline $\mathrm{Z}$ & $*$ & $* *$ & $-99.46 \mathrm{~N} / \mathrm{mm}$ & $\#$ & $\# \#$ \\
\hline$\xi$ & 0 & 0 & $\#$ & $-34 \mathrm{Nm} / \mathrm{rad}$ & 0 \\
\hline$\gamma$ & 0 & 0 & $\# \#$ & 0 & $-34 \mathrm{Nm} / \mathrm{rad}$ \\
\hline
\end{tabular}

Where

$$
\begin{aligned}
& *=-9.79 \mathrm{~N} / \mathrm{mm} \text { at } \mathrm{x}=0.5 \mathrm{~mm} \quad \#=1077.8 \mathrm{Nm} / \mathrm{rad} \text { at } \xi=10^{0} \\
& =-20 \mathrm{~N} / \mathrm{mm} \text { at } \mathrm{x}=1.0 \mathrm{~mm} \quad=1088 \mathrm{Nm} / \mathrm{rad} \text { at } \xi=15^{0} \\
& =-30 \mathrm{~N} / \mathrm{mm} \text { at } \mathrm{x}=1.5 \mathrm{~mm}, \quad=1571 \mathrm{Nm} / \mathrm{rad} \text { at } \xi=20^{0} \\
& * *=-9.79 \mathrm{~N} / \mathrm{mm} \text { at } \mathrm{y}=0.5 \mathrm{~mm} \quad \# \#=1077.8 \mathrm{Nm} / \mathrm{rad} \text { at } \gamma=10^{0} \\
& =-20 \mathrm{~N} / \mathrm{mm} \text { at } \mathrm{y}=1.0 \mathrm{~mm} \quad=1088 \mathrm{Nm} / \mathrm{rad} \text { at } \gamma=15^{0} \\
& =-30 \mathrm{~N} / \mathrm{mm} \text { at } \mathrm{y}=1.5 \mathrm{~mm}, \quad=1571 \mathrm{Nm} / \mathrm{rad} \text { at } \gamma=20^{0} \text {. }
\end{aligned}
$$

\section{CONCLUSION}

A simple mathematical formulation employing the Coulombian model using a vector 
approach is presented for the investigation of forces (radial and axial), moments on the rotor and stiffness of a permanent magnet bearing. Comparison of the results obtained for an axially magnetized permanent magnet bearing with that reported in the literature and 3D FEA shows very good agreement. An attempt has been made to determine the effect of radial and axial displacements of the rotor on force, stiffness and moment in a permanent magnet bearing. It is observed that the effect of radial displacement on force and stiffness is least where as on the moment is quite prominent (magnitude of moment about $\mathrm{X}$ or $\mathrm{Y}$ axis increases with the higher radial offset value). The magnitude of moment increases with an increase in the number of rings in the stack. Full evaluation of the permanent magnet bearing performance is presented by calculating $5 \times 5$ stiffness matrix representing five degrees of freedom. The presented work can be used for optimizing the design of an axially magnetized permanent magnet bearing for wide applications and also, this method involves less computations than the approaches utilizing elliptical integrals.

\section{REFERENCES}

1. Yonnet, J. P., "Passive magnetic bearings with permanent magnets," IEEE Trans. Magn., Vol. MAG-14, 803-805, 1978.

2. Yonnet, J. P., "Permanent magnetic bearings and couplings," IEEE Trans. Magn., Vol. MAG-17, 1169-1173, 1981.

3. Delamare, J., E. Rulliere, and J. P. Yonnet, "Classification and synthesis of permanent magnet bearing configurations," IEEE Trans. Magn., Vol. 31, No. 6, 4190-4192, 1995.

4. Chu, H. Y., Y. Fan, and C. S. Zhang, "A novel design for the flywheel energy storage system," Proceedings of the Eighth International Conference on Electrical Machines and Systems, 1583- 1587, 2005.

5. Guilherme, G. S., R. Andrade, and A. C. Ferreira, "Magnetic bearing sets for a flywheel system," IEEE Trans. on Applied super conductivity, Vol. 17, No. 2, 2150-2153, 2007.

6. Ohji, T., et al., "Conveyance Test by Oscillation and Rotation to a Permanent Magnet Repulsive- Type Conveyor," IEEE Trans. on Magnetics, Vol. 40, No. 4, 3057-3059, 2004.

7. Hussien, A., et al., "Application of the repulsive-type magnetic bearing for manufacturing micromass measurement balance equipment," IEEE Trans. Magn., Vol. 41, No. 10, 3802-3804, 2005.

8. Yonnet, J. P., et al., "Stacked structures of passive magnetic bearings," J. Appl. Phys., Vol. 70, No. 10, 6633-6635, 1991.

9. Ravaud, R., G. Lemarquand, and R. Lemarquand, "Analytical calculation of the magnetic field created by permanent magnet rings," IEEE Trans. Magn., Vol. 44, No. 8, 1982-1989, 2008.

10. Babic, S. I. and C. Akyel, "Improvement in the analytical calculation of the magnetic field produced by permanent magnet rings," Progress In Electromagnetics Research C, Vol. 5, 71-82, 2008.

11. Ravaud, R. and G. Lemarquand, "Comparison of the coulombian and amperian current models for calculating the magnetic field produced by radially magnetized arc-shaped permanent magnets," Progress In Electromagnetics Research, Vol. 95, 309-327, 2009. 
12. Ravaud, R., G. Lemarquand, V. Lemarquand, and C. Depollier, "Discussion about the analytical calculation of the magnetic field created by permanent magnets," Progress In ElectromagneticsResearch B, Vol. 11, 281-297, 2009.

13. Ravaud, R., G. Lemarquand, and V. Lemarquand, "Magnetic field created by tile permanent magnets," IEEE Trans. Magn., Vol. 45, No. 7, 2920-2926, 2009.

14. Selvaggi, J. P., et al., "Calculating the external magnetic field from permanent magnets in permanent-magnet motors - An alternative method," IEEE Trans. Magn., Vol. 40, No. 5, 3278-3285, 2004.

15. Lang, M., "Fast calculation method for the forces and stiffnesses of permanentmagnet bearings," 8th International Symposium on Magnetic Bearing, 533-537, 2002.

16. B. Paden, N. Groom, and J. Antaki, "Design Formulas for Permanent- Magnet Bearings," ASME Trans., Vol. 125, 734-739, 2003.

17. Azukizawa, T., S. Yamamoto, and N. Matsuo, "Feasibility study of a passive magnetic bearing using the ring shaped permanent magnets," IEEE Trans. Magn., Vol. 44, No. 11, 4277-4280, 2008.

18. Samanta, P. and H. Hirani, "Magnetic bearing configurations: Theoretical and Experimental studies," IEEE Trans. Magn., Vol. 44, No. 2, pp. 292-300, 2008.

19. Ravaud, R., G. Lemarquand, and V. Lemarquand, "Force and stiffness of passive magnetic bearings using permanent magnets. Part 1: Axial Magnetization," IEEE Trans. Magn., Vol. 45, No. 7, 2996-3002, 2009.

20. Ravaud, R., G. Lemarquand, and V. Lemarquand, "Force and stiffness of passive magnetic bearings using permanent magnets. Part 2: Radial Magnetization," IEEE Trans. Magn., Vol. 45, No. 9, 3334-3342, 2009.

21. Jiang, W., et al., "Forces and moments in axially polarized radial permanent magnet bearings," Proceedings of Eighth International Symposium on Magnetic Bearings, Mito, Japan, 521-526, 2002.

22. Jiang, W., et al., "Stiffness analysis of axially polarized radial permanent magnet bearings," Proceedings of Eighth International Symposium on Magnetic Bearings, Mito, Japan, 527-532, 2002.

23. Bekinal, S. I., T. R. Anil, and S. Jana, "Force, moment and stiffness characteristics of permanent magnet bearings," Proceedings of National Symposium on Rotor Dynamics, Indian Institute of Technology, Madras, India, 161-168, 2011.

24. Ravaud, R. and G. Lemarquand, "Halbach structures for permanent magnets bearings," Progress In Electromagnetic Research M., Vol. 14, 263-277, 2010.

25. Mukhopadhaya, S. C., et al., "Fabrication of a repulsive-type magnetic bearing using a novel arrangement of permanent magnets for vertical-rotor suspension," IEEE Trans. Magn., Vol. 39, No. 5, 3220-3222, 2003. 